\title{
Measuring and modelling the thermal behavior of LEDs in structural electronics
}

\author{
Esa Hannila \\ Optoelectronics and Measurement \\ Techniques Research Unit \\ University of Oulu \\ Oulu, Finland \\ esa.hannila@oulu.fi
}

\author{
Noora Heinilehto \\ Technical Research Centre of Finland Ltd, \\ VTT \\ Oulu, Finland \\ noora.heinilehto@vtt.fi
}

\author{
Janne Lauri \\ Optoelectronics and Measurement \\ Techniques Research Unit \\ University of Oulu \\ Oulu, Finland \\ janne.lauri@oulu.fi
}

\author{
Kimmo Keränen \\ Technical Research Centre of Finland Ltd, \\ VTT \\ Oulu, Finland \\ kimmo.keranen@vtt.fi
}

\author{
Tapio Fabritius \\ Optoelectronics and Measurement \\ Techniques Research Unit \\ University of Oulu \\ Oulu, Finland \\ tapio.fabritius@oulu.fi
}

\begin{abstract}
Structural electronics consists of printed electronics and silicon-based rigid electronics and load-bearing supporting parts of a device (plastic, glass etc.). One interesting example of structural electronics is large area elements in which light emitting diodes (LEDs) are embedded into the glass laminate. LEDs are used as light sources to create i.e. smart surfaces for the architectural and automotive industry. Once the LEDs are embedded into the structure, they undergo the high temperature conditions and stresses, which are known to have an impact on their lifetime. Many of these aspects are not known for structural electronics. In this study, a thermal simulation model for surface mounted LED on polymer substrate was designed in Comsol Multiphysics -simulation software and the validity of it was evaluated with T3ster measurements. According to measurements, the simulation model is accurate and temperature variations between the simulation and the measurement results was less than 1.0\%. Developed model could be used as a basis for designing the structural LED elements and evaluating their performance characteristics in different user cases.
\end{abstract}

Keywords- printed electronics, hybrid electronics, model verification, junction temperature

\section{INTRODUCTION}

One emerging field of electronics, the hybrid electronics, which is a combination of flexible printed electronics and rigid silicon-based electronics. It is designed to exploit the pros of both techniques to acquire the large area, low-cost and flexibility of printed electronics and the reliability and high performance of semiconductor devices [1-3]. Grown interest have been focused on hybrid electronics because it enables merging the electronics functionalities within the load-bearing, protective device structures, leading eventually to another concept, so called structural electronics [4-5].

This work is financially supported by Academy of Finland's FIRI funding (grant no. 320017) and European Regional Development Fund's Novel Digitally Fabricated Materials for Electronics, Optics and Medical Applications (grant no. A74080).
Smart glasses consisting of electronics embedded within a laminated glass [6-7], an extensively utilized element in architectural industry as enhancing, decorative elements or as a protective windshields of a vehicle in automotive industry [810] are practical examples of structural electronics. In addition, different plastic integrated gadgets and user interfaces have been reported recently [11]. For now, the main interest of industry lies on different type of smart surfaces including i.e. dynamic features displays or Internet of Things (IoT) connectivity. Structural electronics requires efficient and reliable electronics to be merged as part of the bendable or transparent structure where replacement components are not feasible afterwards due its nature. That is why, the material and component selection, the electrical and structural design and used fabrication techniques has to be done carefully to obtain high quality structural electronics products.

Light-emitting devices (LEDs) based on conventional semiconductors, typically silicon, are used as a light sources in optoelectronics based smart surfaces. LEDs are suitable for structural hybrid electronics due their great light emitting properties, low power requirements, small size and long lifetime [12-13]. LEDs are comprehensively studied and well understood in terms of functionality and reliability. The pn-junction temperature and driving current are known to have major impact on their performance and lifetime [14-15]. Since luminosity is also current-dependent, reducing drive-current to lower junction temperature of a devices is not an option. Instead, a proper design for heat transferring structure is necessary to lower the junction temperature.

In the case of structural electronics, the LEDs undergoes considerably high temperature cycle during the manufacturing process. In addition, their operational conditions inside the structural element are unconventional. The effects of those phenomena on LED based structural element performance and 


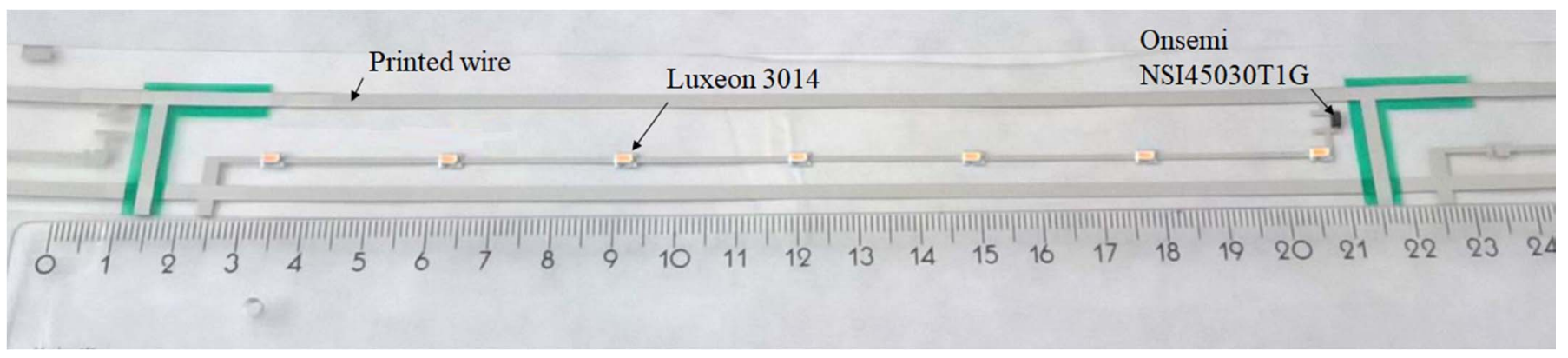

Fig. 1. Photography of the samples measured with T3ster. The results are utilized in evaluation of the Comsol simulation model. Sample's design consists of printed conductive wire pattern and the multiple serially connected LEDs and the regulator. Surface mounted components are attached on the wire patterns and substrate using adhesives. One adhesive is utilized to maintain galvanic contact to the conductors and the second adhesive is utilized to prevent components from detaching as a result of mechanical stresses.

lifetime are not well known. Because the optimization of those aspects, based on manufacturing multiple version of the device to develop the most optimal design for heat transferring structure, is expensive and time consuming, a quicker and more inexpensive modelling based design is required. There are some publicly available simulations for temperature distribution in LED. However, they are made for different material and structural combinations. In addition, existing simulations are created for different purpose and focus on thermal conduction to various heating elements i.e. heat sinks instead of simulating LED's temperature behavior as part of structural electronics where the use of such elements may not be possible i.e. due transparency requirements. [16-18]

In this study we have created a model of Luxeon 3014 LED to simulate its thermal behavior in plastic. Junction temperature of the LED samples are measured, and the results are compared with the simulation results in order to verify the validity and accuracy of the simulation model. We aim to create an accurate simulation model that allows a fast and low-cost means to evaluate the LED's temperature behavior in different environments for various user cases and applications.

\section{Materials AND METHODS}

LEDs are assembled and attached to printed conductive wires and flexible PET film using two adhesives. Identical model, a digital replica is created using Comsol Multiphysics -

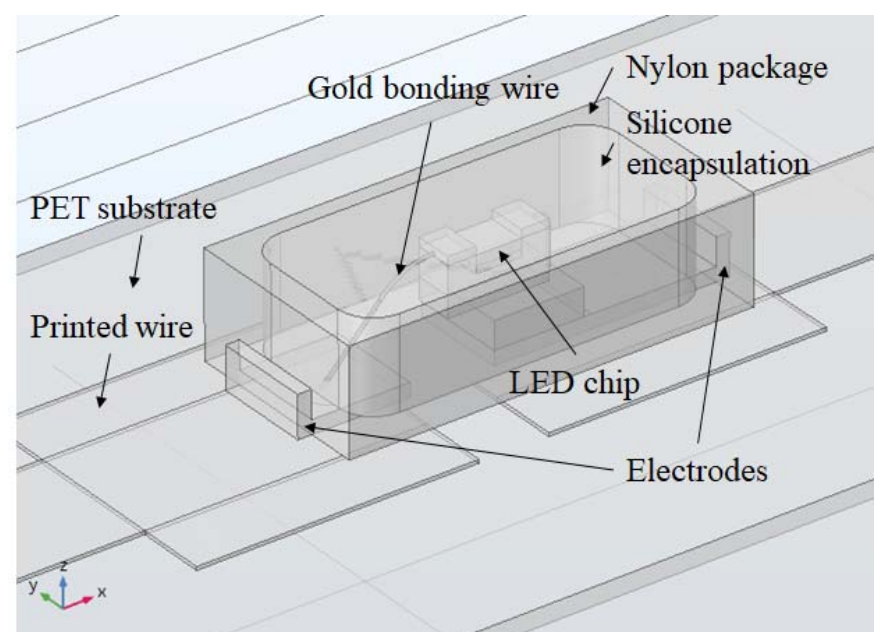

Fig. 2. Photograph of LED simulation model's geometry designed in Comsol Multiphysics -simulation software. simulation software. Measurement results for the sample are utilized to evaluate the simulation model.

\section{A. Specifications of the used materials}

Measured sample structure consists of screen printed conductors on polyethylene terephthalate (PET) substrate with thickness of $125 \mu \mathrm{m}$. LS-411AW silver ink by Asahi is used for the screen printing of conductive wires. Luxeon 3014 LEDs were attached on the substrate and conductors with two types of adhesives. The first adhesive is used to prevent the LED from getting detached from the substrate by mechanical stresses during and after the manufacturing. The second adhesive is used to ensure galvanic contact to the conductors. Materials used in the manufacturing process of the samples and their technical details are summarized in Table I.

\section{B. Layout of the sample}

The layout of the samples is simple printed conductive wire pattern and it is depicted in Fig. 1. In addition to printed silver wires, the sample consists of LED components and a regulator to control fluctuations in supply voltage. There is two parts to the wire pattern printed with two different linewidth. Most of the wire pattern is printed with higher linewidth while only small portion of the wire pattern is printed with lower linewidth. Each component is connected in series within the narrower part of the printed wire pattern.

\section{Fabrication process of the samples}

Fabrication process for the measured samples consists of two main steps. In the first step, the conductive wires are rotary TABLE I

SPECIFICATIONS OF USED MATERIALS AND COMPONENTS

\begin{tabular}{|c|c|c|}
\hline Material/Component & Details & Comment \\
\hline Conductive adhesive & Epotek H20E & $\begin{array}{c}\text { Isotropically } \\
\text { conductive adhesive }\end{array}$ \\
\hline Conductive wires & Asahi LS-411AW & $\begin{array}{l}\text { Silver paste for } \\
\text { screen printing }\end{array}$ \\
\hline Foil & $\begin{array}{c}\text { Polyethylene } \\
\text { terephthalate (PET) }\end{array}$ & $\begin{array}{l}\text { Carrier film for } \\
\text { electronics }\end{array}$ \\
\hline LED & Luxeon 3014 & $\begin{array}{c}\text { Surface mounted } \\
\text { LED }\end{array}$ \\
\hline Regulator & $\begin{array}{c}\text { Onsemi } \\
\text { NSI45030T1G }\end{array}$ & $\begin{array}{l}\text { Surface mounted } \\
\text { regulator }\end{array}$ \\
\hline Support epoxy & Loctite AA 3525 & Supporting epoxy \\
\hline
\end{tabular}


TABLE II

SPECIFICATIONS OF USED MATERIALS IN SIMULATION

\begin{tabular}{|c|c|c|c|c|c|}
\hline Component & Material & $\begin{array}{l}\text { Heat capacity at constant pressure } \\
{\left[\mathrm{J} /\left(\mathrm{KG}^{*} \mathrm{~K}\right)\right]}\end{array}$ & $\begin{array}{l}\text { Density } \\
{\left[\mathrm{kg} / \mathrm{m}^{\wedge} 3\right]}\end{array}$ & $\begin{array}{l}\text { Thermal conductivity } \\
\qquad[\mathrm{W} /(\mathrm{m} * \mathrm{~K})]\end{array}$ & $\begin{array}{l}\text { Electrical conductivity } \\
\qquad[\mathrm{S} / \mathrm{m}]\end{array}$ \\
\hline Electrodes & Copper & 385 & 8960 & 400 & $5.998 \mathrm{e} 7$ \\
\hline Bonding wire & Gold, Au & 129 & 19300 & 317 & $45.6 \mathrm{e} 6$ \\
\hline LED package & Nylon 66 & 1700 & 1150 & 0.28 (User) & $1.0 \mathrm{e}-12$ (User) \\
\hline PET film & PET & 1300 (User) & 1400 (User) & Function & $1.0 \mathrm{e}-3$ (User) \\
\hline Printed Wiring & $\begin{array}{l}\text { Printed } \\
\text { Silver }\end{array}$ & 245 (User) & 2650 (User) & 20 (User) & $2.5 \mathrm{e} 6$ (User) \\
\hline LED chip & Silicon & 700 & 2329 & 130 (User) & $1.0 \mathrm{e}-12$ \\
\hline $\begin{array}{c}\text { LED } \\
\text { encapsulant }\end{array}$ & Silicone & Function & Function & 0.2 (User) & 1e-22 (User) \\
\hline $\begin{array}{l}\text { Conductive } \\
\text { adhesive }\end{array}$ & $\begin{array}{l}\text { Silver filled } \\
\text { epoxy }\end{array}$ & 787 (User) & 2670 (User) & Function & $2.5 \mathrm{e} 5$ \\
\hline
\end{tabular}

Parameters described as 'Function' are obtained from Comsol libraries. Values with (User) tag are obtained from datasheets.

screen printed on the PET substrate in a roll-to-roll (R2R) process at the premises of VTT Oulu Finland. After the conductors are printed, they are cured in temperature based postprocessing for 2 minutes at $140{ }^{\circ} \mathrm{C}$. After the curing of the printed wires, LEDs and regulator are attached. A conductive adhesive, Epotek H20E is utilized to ensure the galvanic contact between the LED electrodes and the printed wire. Since H20E breaks easily when subjected to mechanical stress, a UV-curable supporting epoxy AA 3525 by Loctite is utilized to reinforce the conductive bond by preventing the components from detaching the substrate and printed wires.

\section{T3ster measurements}

T3ster is a measurement device for thermal transient testing. It is utilized in characterization of thermal properties for digital ICs and semiconductors such as LEDs. The measurement process is based on known constant current and measured voltage to define thermal resistivity of a component or material. Measurement is divided in two main phases. In the first phase the $\mathrm{K}$-factor of the component is defined. $\mathrm{K}$-factor is defined by leading a small sensing current, which does not result in heating, through the sample and measuring the voltage drop over the component at certain temperature range. The K-factor is then obtained from the slope of voltage-temperature curve. During the second phase of the measurement, the sample is heated using a heating current. After the temperature of the sample is stabilized the heating current is switched back to sensing current. The voltage over the LED is measured while the sample cools down at ambient temperature. Voltage drop is converted to temperature drop using sensitivity value which is reciprocal value to $\mathrm{K}$-factor defined in first phase. In this study T3ster is utilized to measure thermal properties of the LED by measuring its junction temperature. [19-20]

\section{E. Creating the simulation model}

Simulation model is created using Comsol Multiphysics, which is a finite element analysis, solver and multiphysics simulation software. The heat transfer module is used to analyze the temperature behavior of the simulation model. Measured values for the sample are used as an example to design the geometry of the simulation model (see Fig. 2.). LED is attached to the printed silver wires with conductive adhesive on top of the PET substrate. The LED model consists of silicon LED chip submerged in silicone encapsulation surrounded by a Nylon 66 packaging. LED also has two copper electrodes, which are connected to LED chip with golden wire bonding. All the material parameters used in simulation are presented in Table II. According to simulation results, the distance between adjacent LEDs is long enough to prevent them from affecting each other temperature. Hence, only one LED is simulated in order to keep the time spent on simulation reasonable. Additionally, regulator is removed during the T3ster measurements thus it is missing in simulation as well.

\section{F. Temperature simulation}

LED's temperature behaviour was simulated by setting constant heat rate for active area of the LED. LED's efficiency was measured during the study. Approximately 42,6\% of electrical power is converted to lighting power, whereupon remaining $57,4 \%$ of total electrical power is converted as heating power. According to datasheet of the LED provided by the manufacturer, the forward voltage over LED is $2.83 \mathrm{~V}$ when using the forward current of $25 \mathrm{~mA}$ making total power of 70,8 $\mathrm{mW}$ and, resulting in heating power of $40,6 \mathrm{~mW}$. Heating power is set accordingly and the heat transferring methods, convection and radiation, are defined properly depending on the orientation of the LED. Simulated values for the junction temperatures are then compared with measured temperature values to evaluate the simulation model.

\section{MEASUREMENT RESUltS}

LED samples were measured with T3ster thermal transient testing device, which allows measuring the junction temperature of a LED. Comsol temperature simulation is created to evaluate LED junction temperature in similar manner to measurements. 


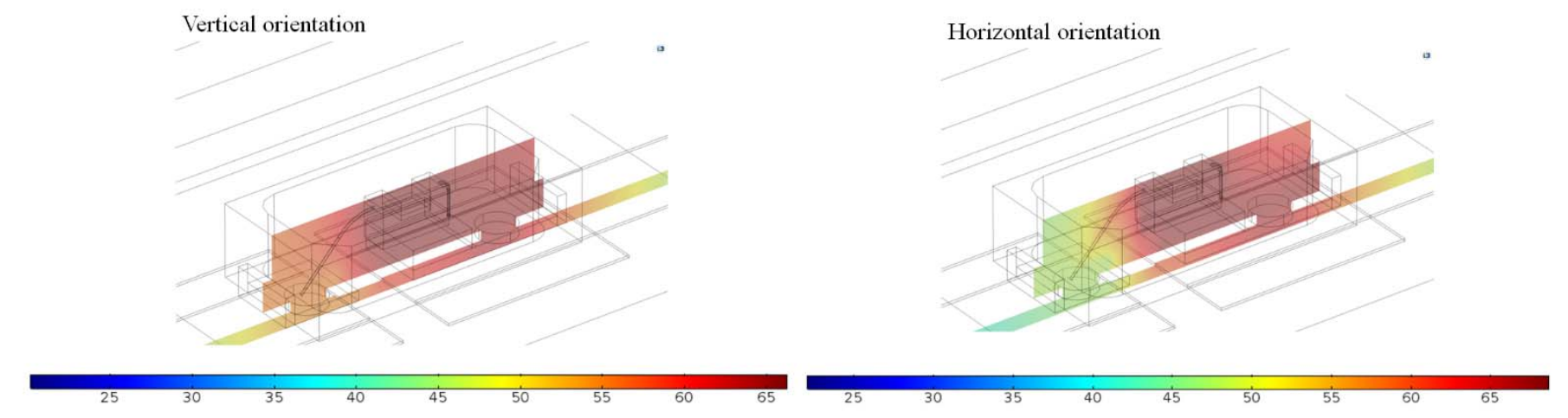

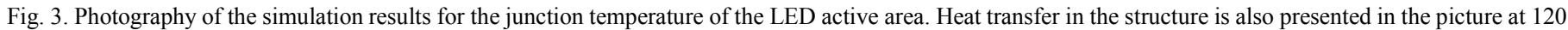

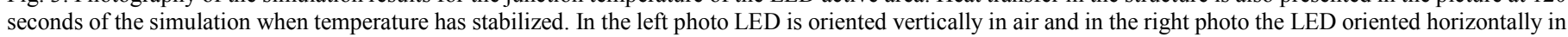
air.

\section{A. T3ster measurements results}

T3ster is utilized to measure the junction temperature of the LED's active area. Altogether there are two separate measurements. At first, LED samples are arranged vertically in air. LED samples are heated by using a forward heating current of $25 \mathrm{~mA}$ while the change in junction temperature is measured. After measuring LEDs vertically, they are measured horizontally by repeating the same measurement process. Measurement results are presented in Table III. LED is heated for 2 minutes during which the junction temperature rises and stabilizes at $68,3{ }^{\circ} \mathrm{C}$ (horizontal) and $66,7{ }^{\circ} \mathrm{C}$ (vertical). According to measurements, the junction temperature of the LED is higher when it is oriented horizontally in air compared to when it is oriented vertically. The horizontal temperature is approximately $2,4 \%$ higher than vertical temperature.

\section{B. Temperature simulation verification}

There are two simulations for the junction temperature as well. In both simulations, a constant heating power is set to correspond $25 \mathrm{~mA}$ heating current. In the first case, the LED is oriented vertically in air while the junction temperature is simulated. The second simulation is identical to first one but the LED is oriented horizontally respectively to measurements. The junction temperature of the LED is measured in the simulation by setting a domain probe for the active area of the LED. Domain probe is a tool in Comsol, which enables measurements for the average value of desired variable of selected domain (in this case temperature). Simulation results are presented in Table III. and in Fig. 3. Temperature rises rapidly during the first 30 seconds for both simulations until it stabilizes at $68,7{ }^{\circ} \mathrm{C}$ (horizontal) and $66,1^{\circ} \mathrm{C}$ (vertical). The orientation of the LED affects on simulated junction temperature similarly to T3ster measurement. While LED is oriented in horizontal direction, its TABLE III

MEASURED RESULTS VS. SIMULATED RESULTS

\begin{tabular}{lll}
\hline \hline \multirow{2}{*}{ Orientation of LED } & Results & $\begin{array}{l}\text { Temperature }\left({ }^{\circ} \mathrm{C}\right), \\
\mathrm{I}=25 \mathrm{~mA}\end{array}$ \\
\hline Horizontal in air & Simulated & 68,7 \\
& Measured & 68,3 \\
Vertical in air & Simulated & 66,1 \\
& Measured & 66,7 \\
& \\
\hline \hline
\end{tabular}

temperature is approximately $3,9 \%$ higher compared to when its oriented vertically.

\section{Simulation case for verified model}

We also created a third simulation for the LED model to demonstrate a case in which LED is embedded inside the 3D printed plastic. In this simulation, the LED model is embedded inside a polylactide (PLA) block, which is common filament material used in 3D printing technology. There is an air gap between the PLA structure and LED, which forms a $50 \mu \mathrm{m}$ thick insulator layer. The efficiency of the LED is assumed as constant within the temperature range during the simulation and the same heating power is used as in earlier simulation. Simulation results are presented in Fig. 4.-5. Stabilization of the temperature now takes much more time and the temperature rise higher than it did in air. After 30 minutes of the simulation, the temperatures of

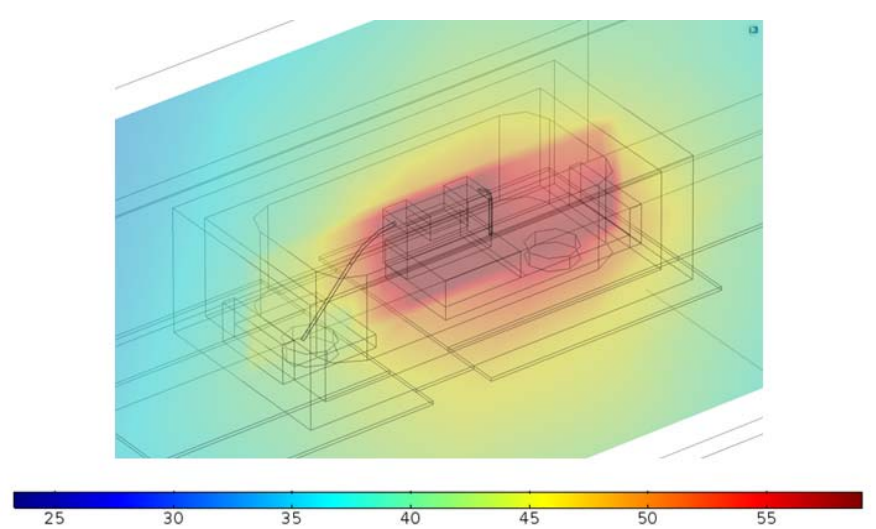

Fig. 4. Photography of the simulation results for the junction temperature of the LED that is embedded inside PLA structure. Small air gap between the LED and PLA structure act as $50 \mu \mathrm{m}$ thick insulator layer affecting the junction temperature. 


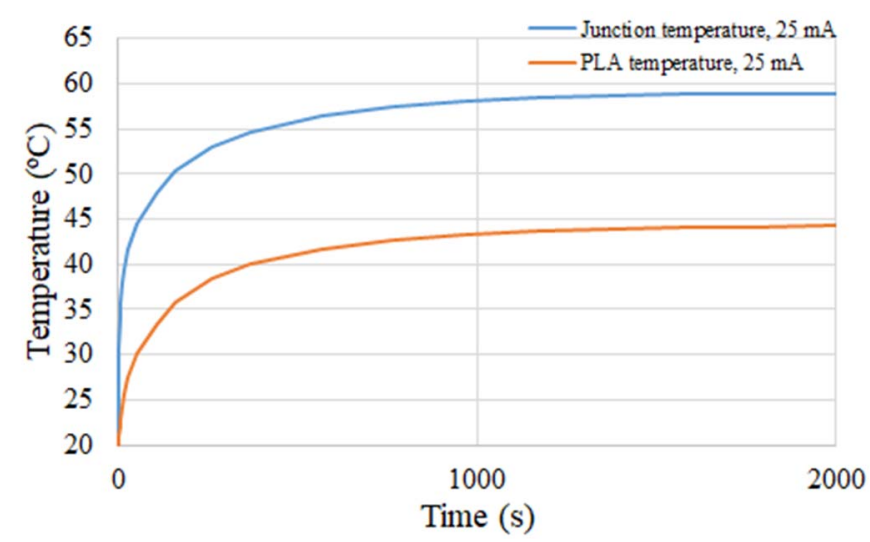

Fig. 5. Graph of the LED's junction temperature and the PLA surface next to it as a function of time. Temperature rises higher and stabilizes more slowly than it did in air.

the LED and the PLA surface around it have risen approximately to $58,9^{\circ} \mathrm{C}$ and $44,3{ }^{\circ} \mathrm{C}$.

\section{DISCUSSIONS AND CONCLUSION}

As shown in Fig. 3 the highest heat elevation is located at the pn-junction of the LED as it should. Heat conducts through the structure to the surface from which the heat transfer is both convective and radiative. Performed simulation and the independently conducted measurement results are almost identical verifying the accuracy of the proposed model. When LED is oriented horizontally, simulated temperature value is approximately $0,6 \%$ above the measured value and $0,9 \%$ below the measured value when LED is oriented vertically (See Table III). In both measured and simulated results, the junction temperature was higher when LED is oriented horizontally in air than when it was oriented vertically. This phenomenon can be explained by the improved heat convection of vertical orientation.

Small differences in simulated and measured junction temperatures can be partially explained by the geometry and material properties used in the simulation model. Some geometry related features were not created with an absolute accuracy since every detail from the structure of the LED is not available. Hence, the model's geometry is simplified version and involve inaccuracies. Additionally, manufacturers may utilize material compounds with enhanced material properties such as thermal conductivity i.e. Nylon 66. Every detail of material parameter may not be available or they might variate depending on the material manufacturer. Assumptions of those compounds may result in inaccurate material parameters and simulation results.

As the simulation model was found to be valid, a third simulation was made to investigate thermal characteristics of the LED element inside a 3D printable plastic cover. According to results, the junction temperature does not rise as high as it does in air, even if the convective heat transfer for LED is nonexistent in such structure and a small, thermally insulating air gap is existing between the LED and PLA structure. Since the gap size is small, the lower junction temperature is likely to be explained by the improved heat conduction to PLA structure. However, exceeding the glass transition temperature (GTT), which is approximately $60{ }^{\circ} \mathrm{C}$ for PLA, should be avoided. Currently, the surface temperature for PLA surfaces surrounding the LED is less than $16^{\circ} \mathrm{C}$ below the GTT even with the lowest operating conditions, emphasizing the importance of proper design rules and right material and component selection. In addition, possible LED efficiency variations was kept constant, which is not the case in reality. Another concern for such structure would be thermomechanical stress, which might cause some problems but is not yet evaluated and is natural continuation for the simulation.

Existing model is verified at single point but in order to make it more reliable, additional measurements with higher currents and taking the temperature and current-dependent efficiency and thermomechanical stresses into account is needed. Since the lifetime reliability of a LED depends on junction temperature and forward current, extended simulation model could also be utilized for evaluating the reliability related aspects enabling the product lifetime analysis for different user cases.

\section{REFERENCES}

[1] G. Tong, Z. Jia and J. Chang, "Flexible Hybrid Electronics: Review and Challenges," in Proceedings - IEEE International Symposium on Circuits and Systems, Florence, 2018.

[2] Y. Khan et al., "Flexible Hybrid Electronics: Direct Interfacing of Soft and Hard Electronics for Wearable Health Monitoring," Advanced Functional Materials, vol. 26, no. 47, pp. 8764-8775, 2016.

[3] K. Remes and T. Fabritius, "Non-contact Characterization of Flexible Hybrid Electronics by Synchronized Thermography," IEEE Transactions on Instrumentation and Measurement, no. DOI: 10.1109/TIM.2019.2962576, 2019.

[4] E. MacDonald et al., "3D printing for the rapid prototyping of structural electronics," IEEE Access, vol. 2, pp. 234-242, 2014.

[5] A. Lopes, E. MacDonald and R. Wicker, "Integrating stereolithography and direct print technologies for 3D structural electronics fabrication," Rapid Prototyping Journal, vol. 18, no. 2, pp. 129-143, 2012.

[6] J. Lauri, E. Hannila and T. Fabritius, "Non-destructive characterization of glass laminated electronics," in Conference Record - IEEE Instrumentation and Measurement Technology Conference, Auckland, 2019.

[7] E. Hannila, B. Augustine, T. Kurkela, J. Lauri and T. Fabritius, "Yield and Electrical Functionality of the Glass Laminated Conductive Wires and Connectors," IEEE Transactions on Components, Packaging and Manufacturing Technology, vol. doi: 10.1109/TCPMT.2019.2923858.

[8] J. Xu, Y. Li, B. Liu, M. Zhu and D. Ge, "Experimental study on mechanical behavior of PVB laminated glass under quasi-static and dynamic loadings," Composites Part B: Engineering, vol. 42 , no. 2, pp. 302-308, 2011.

[9] A. Berenjian and G. Whittleston, "History and Manufacturing of Glass," Americal Journal of Material Science, vol. 7, no. 1, pp. 18-24, 2017.

[10] A. Vedrtnam and S. Pawar, "Laminated plate theories and fracture of laminated glass plate - A review," Engineering Fracture Mechanics, vol. 186, pp. 316-330, 2017.

[11] T. Kololuoma et al., "Adopting Hybrid Integrated Flexible Electronics in Products: Case - Personal Activity Meter," IEEE Journal of the Electron Devices Society, vol. 7, pp. 761-768, 2019.

[12] M. Raypah, M. Devarajan and F. Sulaiman, "Effects of Substrate on Thermal and Optical Characteristics of High-Power ThinGaN White LED," IOP Conference Series: Materials Science and Engineering, vol. 409, no. 1, 2018.

[13] T. Cheng, X. Luo, S. Huang and S. Liu, "Thermal analysis and optimization of multiple LED packaging based on a general analytical 
solution," International Journal of Thermal Sciences, vol. 49, no. 1, pp. 196-201, 2010

[14] Lumileds, "WP15 Evaluating the Lifetime Behavior of LED Systems White Paper 20161201," Lumileds, 1 December 2016. [Online]. Available: https://www.lumileds.com/uploads/167/WP15-pdf. [Accessed 20 September 2019].

[15] H. Tang et al., "Multi-physics simulation and reliability analysis for LED luminaires under step stress accelerated degradation test," in 13th International Thermal, Mechanical and Multi-Physics Simulation and Experiments in Microelectronics and Microsystems, EuroSimE 2012, Cascais, 2012.

[16] O. Tapaninen, J. Ollila, E. Juntunen and K. Keränen, "Thermal management in flexible substrates for LEDs," in IMAPS Nordic Annual Conference Proceedings, Oulu, 2014.

[17] H.-K. Fu et al., "Evaluation of temperature distribution of LED module," Microelectronics Reliability, vol. 53, no. 4, pp. 554-559, 2013.

[18] E. Juntunen et al., "Thermal performance comparison of thick-film insulated aluminum substrates with metal core PCBs for high-power LED modules," IEEE Transactions on Components, Packaging and Manufacturing Technology, vol. 2, no. 12, pp. 1957-1964, 2012.

[19] Lumileds, "WP23 How to Measure Thermal Resistance of LED Emitters and LED Arrays White Paper 20161201," Lumileds, 1 December 2016 . [Online]. Available: https://www.lumileds.com/uploads/568/WP23-pdf. [Accessed 20 September 2019].

[20] J. Hu, L. Yang, W. Hwang and M. Shin, "Thermal and mechanical analysis of delamination in GaN-based light-emitting diode packages," Journal of Crystal Growth, vol. 288, no. 1, pp. 157-161, 2006. 